\title{
Coulomb explosion in nanosecond laser fields
}

\author{
Jie Zhang, Yuzhong Yao, and Wei Kong* \\ Department of Chemistry, Oregon State University, Corvallis, OR 97331
}

Supplementary document

To be submitted to the Journal of Journal of Physical Chemistry Letters, January 9, 2020.

*Corresponding author, wei.kong@oregonstate.edu, 541-737-6714 (ph), 541-737-2062 (fax) 


\section{Measurements of cluster sizes}

In this experiment, the time-of-flight mass spectrometer is rearranged to be in-line with the direction of the cluster beam. At $400 \mathrm{~K}$, the average speed of an argon atom is $500 \mathrm{~m} / \mathrm{s}$. Based on our simulation, the effect of this speed on the cluster size is a reduction in the resulting size: for a cluster of 100,000 atomic units (u, Ar2500), the actual size of the cluster should be $10 \%$ higher than that measured from the experiment.

To ensure detection of large argon clusters, a high repeller voltage is needed. An argon cluster containing 1000 atoms has a mass-to-charge ratio of 40,000 u. The response function of a standard microchannel plate diminishes quickly to zero with increasing ion masses above $1000 \mathrm{u}$. We varied the voltage on the repeller electrode from $2 \mathrm{kV}$ to $10 \mathrm{kV}$. Although the intensities of the cluster peaks increased with increasing repeller voltage, the resulting cluster sizes did not change. We therefore performed all measurements at $2 \mathrm{kV}$. The signal-to-noise ratio was not ideal, but the experiment was much safer to operate.

A concern with this type of mass spectrometry for cluster size measurement is the possibility of fragmentation, so the resulting size is always considered a lower limit, even if the detector has a uniform response function to the cluster size. We found that if we maintain the laser intensity below $10^{6} \mathrm{~W} / \mathrm{cm}^{2}$, non-resonant two photon ionization at $266 \mathrm{~nm}$ results in minimal fragmentation at least for the compounds investigated in this work. Fig. S1(a) shows the mass spectra recorded under these conditions at two different stagnation pressures, and Fig. S1(b) shows the expanded region containing the clusters under a series of stagnation pressures. 

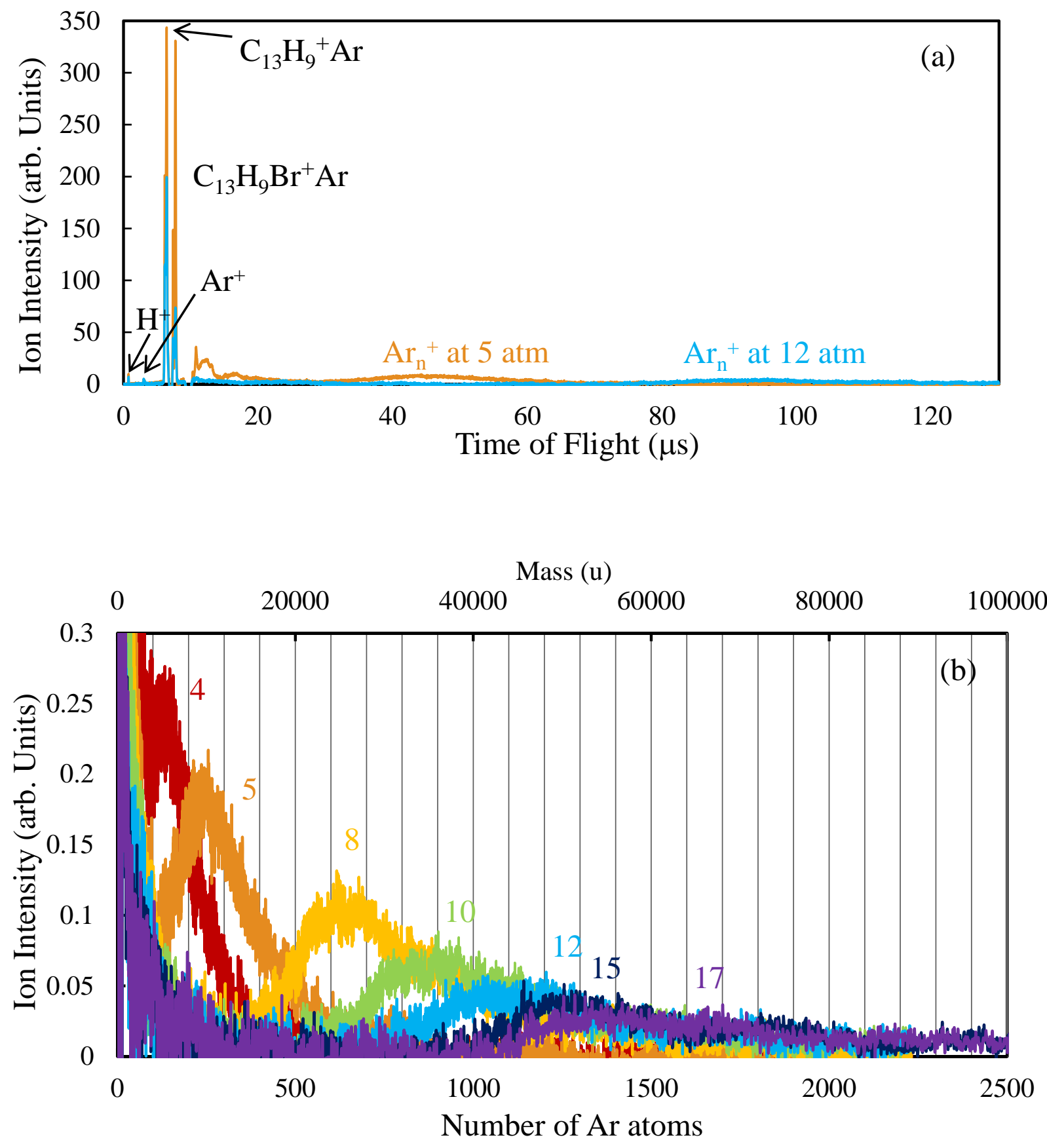

Figure S1. Mass spectra of 2-bromo-fluorene doped argon clusters recorded at $10^{6} \mathrm{~W} / \mathrm{cm}^{2}, 266$ $\mathrm{nm}$, and a vapor pressure of $51 \mathrm{~Pa}$. Panel (a) shows the overall spectra at two different stagnation pressures, and panel (b) shows the expanded view of the cluster portion of the spectra. The stagnation pressures in units of atm are labeled in the figure, colored coordinated with the traces. 
From Fig. S1, the cluster size distribution is broad, and becomes broader with increasing stagnation pressures. In Table S1, we list the most probable size corresponding to the peak of the size distribution and the upper quartile of the distribution.

Table S1. Sizes of $\mathrm{Ar}_{\mathrm{n}}$

\begin{tabular}{c|c|c}
\hline $\begin{array}{c}\text { Stagnation } \\
\text { Pressure }\end{array}$ & $\begin{array}{c}\text { Most } \\
\text { Probable }\end{array}$ & $\begin{array}{c}\text { Upper } \\
\text { Quartile }\end{array}$ \\
\hline 4 & 147 & 290 \\
\hline 5 & 273 & 455 \\
\hline 6 & 478 & 747 \\
\hline 8 & 675 & 1033 \\
\hline 10 & 960 & 1540 \\
\hline 12 & 1156 & 1812 \\
\hline 15 & 1272 & 2432 \\
\hline 17 & 1349 & 2927 \\
\hline
\end{tabular}




\section{Power dependence of MCAI}

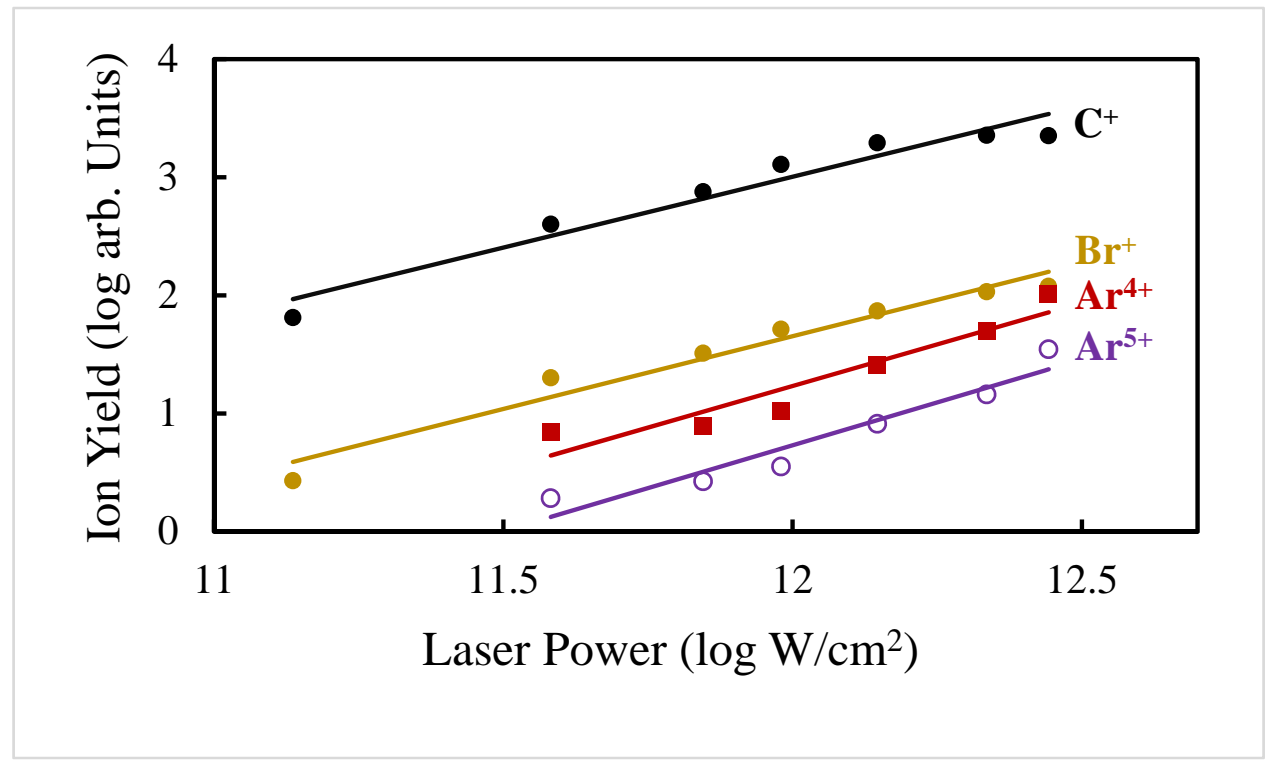

Fig. S2. Power dependence of a few selected atomic ions in a log-log plot. The slopes of the fittings are between 1.2 and 1.4. To produce $\mathrm{Ar}^{5+}$ from $\mathrm{Ar}^{4+}, 33$ photons at $532 \mathrm{~nm}$ are needed. As explained in the main text, these slopes can only be considered as indications of nonlinear and highly saturated regimes of the experiment.

\section{Elimination of a possible artifact of the experiment}

We have considered a possible artifact of the intense extraction field in the ionization region. The photoelectrons could be energized by the strong field, or they could collide with the electrode and produce secondary high energy electrons, and ultimately the energetic electrons can collide with a nearby argon cluster and produce MCAI. Although the fact that no MCAI was observable at $266 \mathrm{~nm}$ under the same settings of the electric field strongly disputes this possibility, we performed another experiment to eliminate this possibility. The extraction field was delayed by 
up to $1 \mu$ s after laser excitation, allowing all photoelectrons to escape the ionization area. The MCAI remains in the mass spectrum after the application of the electrical pulse. 